\title{
An agent-based approach for strategic alignment in enterprise systems: A cancer care case
}

\author{
Mehmet E. Aydin*,1 \\ Software Engineering \\ Research Group \\ University of the West \\ of England \\ Bristol, UK
}

\author{
Ercan Oztemel ${ }^{2}$ \\ Dept. of Industrial \\ Engineering \\ Marmara University \\ Istanbul, Turkey
}

\author{
Dana Nashawati ${ }^{3}$ \\ King Hussain Cancer \\ Center \\ Amman, Jordan
}

\author{
Mohammed Odeh ${ }^{4}$ \\ Software Engineering \\ Research Group \\ University of the West \\ of England
}

Bristol, UK

\begin{abstract}
Enterprise systems are viewed as large-scale software systems, which are usually assembled through a number of protocols inspired by political coalitions. This is due to the fact that incompatibilities emerge through the nature of subsystems can only be avoided in a wider and comprehensive context, which can only come to existence in the corporate/strategic level of enterprises. Cancer care systems consist of a number of such individual systems that require alignment of principles and goals subjected to changes due to emerging circumstances in order to keep up with substantial efficiency, and a certain level of sustainability. In this position paper, an agent-based system is proposed comprising a set of autonomous and collaborating agents running a mathematical model to health-check the current status of the entire system and to produce maintenance and alignment operations to corresponding parts of the system. It helps align the strategical values and principles with the lower level operations infiltrating into the entire enterprise system. This prototypical idea can be extended for more configuration and maintenance.
\end{abstract}

Index Terms-Cancer Care Informatics, Agent-based Computing, Strategic Alignment, Enterprise Systems

\section{INTRODUCTION}

Business in enterprises can always face challenges emerging from the markets and wider ecosystems. Inevitably, this forces enterprises to adapting the changes and introduce new approaches to handle issues for sustainability and enhancement [1]. It is known that managerial and operational activities in enterprise-level organisations are viewed and handled in three levels; strategic, tactic and operational levels. In strategic level, long-term principles and all enterprise-level activities are envisaged and planned ahead for a longer spanning time, while tactic and operational activities are for midterm or shorter time periods and more specific to particular operations and processes.

Change in ecosystem of enterprises is handled in strategic level, which help lead and drive the corporate business into the future. Principles, rules, and targets can be revised periodically or upon any emerging circumstances, but, it is not easy to infiltrate into every single process and operation of the organisation. This is due to the scale and complexity of

\footnotetext{
*Corresponding author

${ }^{1}$ mehmet.aydin@uwe.ac.uk

${ }^{2}$ eoztemel@marmara.edu.tr

${ }^{3}$ dnashawati@khcc . jo

${ }^{4}$ mohammed.odeh@uwe.ac.uk

5 amansour@khcc. jo
}

organisations. Any such change envisaged by the lead of the enterprises is required to be communicated and adopted by the whole body of the organisation so that all can sing the same melody.

A number of studies have been conducted in this regard with respect to various aspects of strategic alignment. Morrison et.al. [2] and Rashidirad et. al. [3] have overviewed strategic alignment fundamentals, concepts and principles for generic business environments while Damiani et. al. [4] and Henderson and Venkatraman [5] propose substantial frameworks to conduct alignment of strategies. On the other hand, Ivanov [6] has introduced a study for aligning changes in strategic plans across a supply chain using an adaptive framework, while Shirazi and Sooror [7] used an agent-based framework to handle issues of strategic information systems in enterprises. Kearns and Subherwal [8] attained strategic alignment in information technologies discussing behaviours, outcomes and concepts within this context. Meanwhile, Avison et.al [9] theoretically evaluate strategic alignment models. To the best knowledge of the authors, neither of the studies mentioned above nor of any unlisted one have attempted modelling strategic alignment process using teams of autonomus agents (i) to screen the health-check of the enterprise, (ii) to repair identifed deficiencies according to setup goals and targets, and (iii) to maintain the complete enterprise system fit to the strategies. In this position paper, a mathematical model has been developed to screen the health-check status of any enterprise subject to any change given rise. In addition, an autonomous system based on multi-agent systems is proposed to monitor the whole enterprise in this respect and maintain the entire corporate solution (systems) automatically. A discussion around a candidate case study of cancer care is provided for prospective implementation.

The rest of this paper is organised as follows: Section II provides an overview on what an enterprise system is, how strategic alignment works within the enterprises and why enterprise systems require strategic alignment. Section III introduces a diagnostic mathematical model to monitor the complete system and discover the deficiencies among subsystems of enterprise systems while Section IV recommends an agent-based alignment process to keep the system up-to-date to achieve strategic homogeneity across the enterprise. Section V briefs a cancer care systems and it potantial for implementation while Section VI includes conclusions. 


\section{Strategic Allignment in Enterprise Systems}

Enterprise systems are large scale software systems in which a number of subsystems, such as components, services and standalone systems, take part of the existence and contribute in a designed capacity. A team of experts from Carnegie Mellon University have authored a report on the size and scale of enterprise systems providing a concise review in various aspects and issuing a vision for the future [10]. Another point of view is made by Sommerville et al [11] suggesting to recognise them as the coalition of systems, where each system plays its-own role based on the capacity and capabilities through its purpose of being. It is conceivable that each independent system that take part of a larger system may not fully match the principles of the other parties and may have some compatibility issues to some extent, but, these issues are resolved with some sort of protocols and agreement similar to political coalitions. Obviously, the incompatibilities and proprietary principles of subsystems would cause clashes and collisions to some extent and will seriously need collaboration built among them to form up larger harmonious systems.

Strategic alignment is one of key concerns in large-scale enterprise systems, is related to system of systems ( $\mathrm{SoS})$, where a unified overall view across the whole enterprise is crucial for harmony and success. As indicated above, a typical enterprise can be viewed and evaluated in three levels; strategic, tactic and operational levels. A complete enterprise system includes a number of functions, processes, values, rules and evaluations criteria associated to the activities, where rules, values, principles and metrics are mostly coupled with strategic level, while functions, operations and processes are more about tactic and operational levels. Sets of tasks make up processes, multiple processes would take part each individual function and the functions would be part of the overall business.

The overall business uses qualities to measure the level of achievements, if the enterprise functions, processes and tasks are all complient to the values, rules and principles. The qualities are measured with a number of key-performance indicators (KPI) in the strategic level, while the processes are evaluated with a different set of metrics, so-called process performance indicators (PPI). A KPI is related to one or more PPIs. Common deficiencies and irregularities include processes missing PPI, non-aligned/interlinked KPI-PPI, nonup-to-date principles and/or rules, change in corporate targets, introduction of new performance metrics, sunsetting selected old ones etc. All clearly require (i) to be identified, (ii) prioritised and (iii) rectified and maintained accordingly.

Fig. 1 sketches a logical topology to interrelate functions, processes and tasks, where a hierarchical relationship is envisaged. Apparently, activity $F$ is made of three distinctive processes while each process comprises different number of tasks (operations). Each of these processes are evaluated/assessed with predefined qualities, $P P I_{\{i, j, k\}}$ as indicated in Fig. 1, which are accounted for the performance level achieved for a particular activity, while enterprise-level qualities are

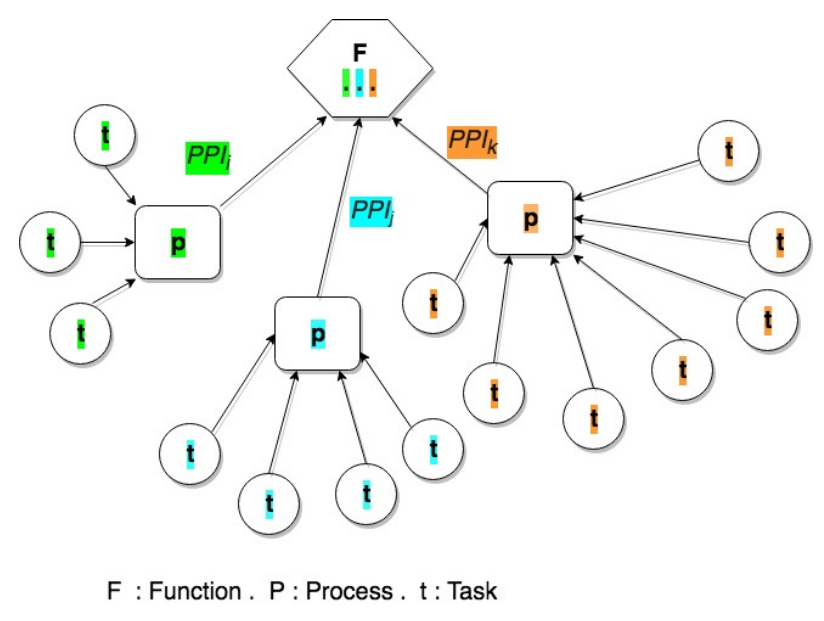

Fig. 1. A logical topology to interrelate functions, processes and tasks in an enterprise environment

accounted delibrating every activity as function across the corporate business. The extended logic of this entire process is modelled in the following section encompassing the entire enterprise accounting all activities.

\section{Modeling Strategic Alignment in Business PROCESSES}

\section{A. The process of strategic alingment}

The process of strategic alignment is outlined in Fig. (2), where the interactions among the major components are reflected in a cyclic form. The main starting part is the strategies in which all the values, principles and key business rules are constituted. The strategic level goals, which are measured with KPIs, are derived to map corresponding values, rules and principles into KPIs. Then, these strategically mapped goals are aligned to the processes and the process level targets with which the performance of each individual process is determined. A set of adjustment and calculative actions help derive the performance of individual workforce staff, afterwards, and then the ultimate system output is concluded. The cycle is completed with a set of analysis to check how strategies are implemented and if there is any misfit in the real ground of the business.

The right-hand side of the cyclic transition process is about strategic alingment process while the left-hand side is more about performance analysis and the level of strategic fitness. The scope of this study is going to cover the right-hand side of the diagram, but will incorporate to the left-hand side componenets with a set of assumptions.

\section{B. Screening model for system health-check}

This subsection intorduces a model suggesting a generic screening and diagnostic set of rules in a more systematic form. It would fit in the circumstances of many enterprises with the generic nature, but, might not consider details specific to particular cases. A bespoke model can always be derived to embrace the enterprise-specific characteristics, which require to be taken under consideration. 


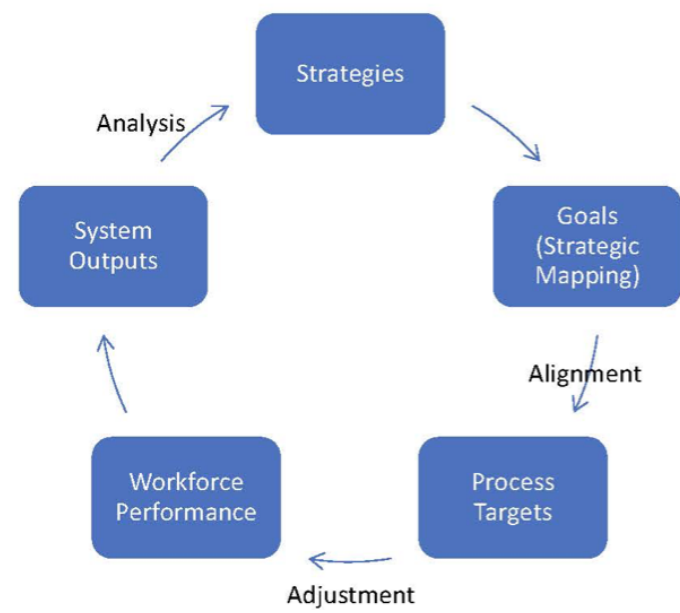

Fig. 2. The stages of strategic alignment process

Given a number of preset strategic goals/targets, an enterprise intends to link all high level principles and business rules to operation-level processes to create a harmony within the entire enterprise so as to improve the efficiency through the processes. Let an enterprise adopt a set of strategic goals, $\mathcal{G}=\left\{g_{t} \mid t=1, . ., T\right\}$, where each particular goal $g_{t}$ is expected to be mapped with at least one key performance indicator (KPI), $\mathcal{K}=\left\{\kappa_{k} \mid k=1, \ldots, K\right\}$, identified to measure the success of the enterprise within a particular time period. Let $\mathbf{x}_{t}=\left\{x_{t, k} \mid t \in T, k \in K\right\}$ be a set of binary variables to indicate whether the KPIs are mapped with any particular strategic goal, where $x_{t, k}$ will take value of 1 if $\kappa_{k}$ is mapped with $g_{t}, 0$ otherwise. The following inequality makes sure that each strategic goal is mapped to at least one KPI.

$$
\sum_{k=1}^{K} x_{t, k} \geq 1 \quad \forall t \in T
$$

Another requirement to meet is that each KPI measure, $\kappa_{k}$, must satisfy corresponding pre-set goal values from a set of values, denoted with, $\mathcal{B}=\left\{\beta_{k} \mid k=1, \ldots, K\right\}$. Here, the constraint can be expressed as:-

$$
\kappa_{k} \geq \beta_{k} \quad \forall k \in K
$$

Following mapping the strategic goals with corresponding KPIs, the operational-level processes should also be considered for performance measurement linking the operationallevel performance indicators and pre-set process/operation goals. Let $\mathcal{A}=\left\{a_{i}|i \in| \mathcal{A} \mid\right\}$ be the set of activities while $\mathcal{P}=\left\{p_{j}|j=1, \ldots,| \mathcal{P} \mid\right\}$ be existing set of processes across the enterprise and $\mathcal{F}_{i}=\left\{f_{i, j}|i \in| \mathcal{P}|, j \in| \mathcal{F}_{i} \mid\right\}$ be the set of operations/functions forming each process ordered and particularly organised. Each process is monitored and measured with another set of performance indicators, so-called process performance indicator (PPI) and denoted with $\pi=\left\{\pi_{i} \mid i=\right.$ $1, \ldots,|\mathcal{P}|\}$. Each process must be mapped with at least one of these, $\pi_{i}$, so that the performances can be indicated. In order to assure that each process must be linked with at least one PPI, a binary variable, $\mathbf{y}_{i}=\left\{y_{i, j}|i \in| \mathcal{P}|, j \in| \mathcal{A}_{i} \mid\right\}$, is used to incorporate this fact into the model, where $y_{i, j}$ will take value of 1 if $\pi_{j}$ is mapped with $p_{i}, 0$ otherwise..

$$
\sum_{j=1}^{\left|\mathcal{A}_{i}\right|} y_{i, j} \geq 1 \quad \forall i \in|\mathcal{P}|
$$

Similar to strategic level, each PPI imposes a target value to be satisfied. Let $\Delta=\left\{\delta_{i}|i \in| \mathcal{P} \mid\right\}$ be the set of target values for PPIs. The following constraint imposes that the corrresponding PPI should remain within the boundaries of the target values.

$$
\pi_{i} \geq \delta_{i} \quad \forall i \in|\mathcal{P}|
$$

The final stage of linkage is to relate KPIs with PPIs with the following union relationship:-

$$
\kappa_{i}=\bigcup_{j=1}^{\left|\kappa_{i}\right|} \kappa_{i, j} \quad \forall i \in K
$$

where $\kappa_{i, j}$ is the $j^{t h}$ component of $i^{t h} \mathrm{KPI}, \kappa_{i}$, and $\left|\kappa_{i}\right|$ is the size of the set of components form up $\kappa_{i}$. These component set can be a sub-set of PPIs, which would be filtered from the complete set of PPIs, $\mathcal{P}$. Therefore, these components will be selected by the means of the binary variable, v. Depending on the way in which the PPI are united, the relationship can turn to the following summation function.

$$
\kappa_{i}=\sum_{j=1}^{|\mathcal{P}|} \pi_{j} v_{i, j} \quad \forall i \in K
$$

where $v_{i, j} \in \mathbf{v}$ is a binary variable identifies if the $j^{\text {th }}$ PPI, $\pi_{j}$, takes part of the $i^{t h} \mathrm{KPI}, \kappa_{i}$, implies that $v_{i, j}=1$ if it takes part, $v_{i, j}=0$ otherwise. This applies to all KPIs, which is a strong leverage to align strategic goals to operational level activities.

The complete model works to check whether or not the set of constraints, $\mathcal{C}=\left\{c_{i}|i=1, . .,| \mathcal{C} \mid\right\}$, satified, where $|\mathcal{C}|=$ $T+K+2|\mathcal{P}|$. As seen, each constraint, $c_{i}$, in the model is in the form of either an equation of inequality made of a right-hand side part, $c_{i}^{r}$, and a left-hand side, $c_{i}^{l}$, part, where the left-hand side parts represent the formal structure of the rules with variables and parameters and right-hand side part presents the actual planned and targeted data and information level.

The level of satisfaction for the complete model is measured constraint-by-constraint, and accumulated, accordingly, through Eq. (8) and (7). For the purpose of aggrigating all constraints into the model, a set of binary indicators, $D=\left\{d_{i}|i \in| \mathcal{C} \mid\right\}$, is used to reflect if a particular constraint, $c_{i}$, is satisfied.

$$
d_{i}= \begin{cases}1 & \text { if }\left(c_{i}^{l}-c_{i}^{r}\right)<0 \quad \forall i \in|\mathcal{C}| \\ 0 & \text { otherwise }\end{cases}
$$


and,

$$
\operatorname{Min} \quad Z=\sum_{i=1}^{|\mathcal{C}|} d_{i}
$$

where evaluation of the constraints is conducted as the difference between the right-hand side, $c_{i}^{r}$ and left-hand side, $c_{i}^{l}$. Since it is expected that the left-hand side, $c_{i}^{l}$, is to be greater than or equal to the right-hand-side, $c_{i}^{r}$, the difference, $\left(c_{i}^{l}-c_{i}^{r}\right)$, should be at least equal to 0 , which is the desirable value in order to keep the system minimised of constraint violation.

This diagnostic model consists of a number of constraints, which check for any deviations between targeted values and those on the ground, and an objective of minimising the deviations from the targets. It makes the model robust and relient on firm grounds as it can easily be expressed in a constraint satisfaction model, which is one of very well-known NP-hard combinatorial optimisation models.

\section{An Agent-Based Problem Solving Approach}

The strategic alignment problems across enterprises are to be handled and solved using an agent-based approach in which a number of roles are identified and played by individual agents, collaboratively for high efficiency. The agent based system is expected to supply a set of autonomous virtual entities that act independently and proactively to explore the best time for acting to diagnose, repair and maintain accordingly. The main gain would be not to engage the IT systems of the corporate during prime time, but explore the best time to act using autonomy granted to the agents. This is clearly not be possible with non-autonomous approaches. Besides, distributed intelligence over the agent helps harness the capabilities of the agents not all the time, but, upon necessity. Agent-based approaches and multi agent systems have been used successfully by many studies in various domains including dynamic scheduling [12], collaborative job scheduling [13]. Particularly, change management [14] presents a multiagent approach for change management in manufacturing enterprises, which involve very-large-scale enterprise systems. This work is very highly inspiring for change management of enterprise systems in other industries such as health-care systemns.

Agent-based systems have also been used for extending capabilities of swarm intelligence algorithms [15], incorporating with machine learning approaches [16]. Ivanov [6] has introduced a study for aligning changes in strategic plans across a supply chain using an adaptive framework, while Shirazi and Sooror [7] used an agent-based framwork to handle issues of strategic information systems in enterprises.

The problem solver in this approach is deviced based on a multi-agent approach, which is organised following the workflow required by the process. Fig. (3) depicts the framework of the multi agent system designed to solve strategic alignment problem explained and model in the previous section. As the major components of the system, a business process model (BPM), which includes the formulations and relevant business

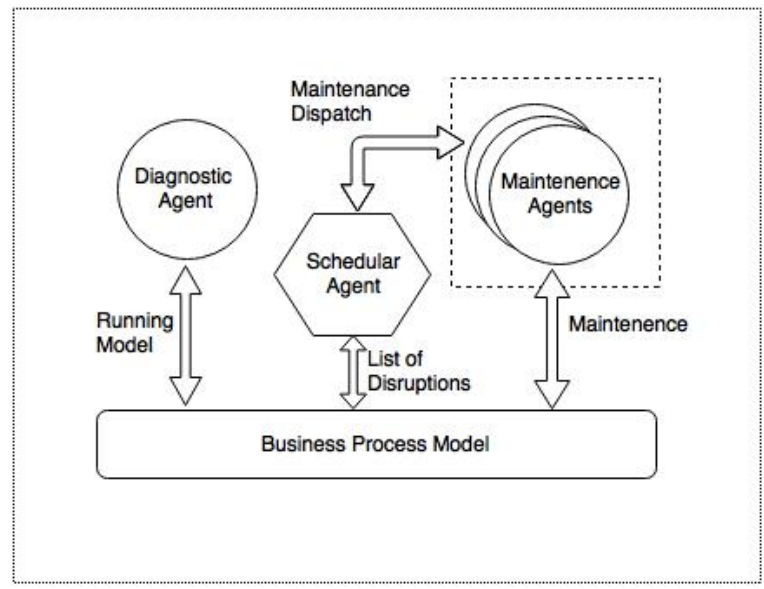

Fig. 3. The multi-agent system devised to diagnose and repair/maintain business process model

rules, accordingly. BPM is the main source of information and knowledge fed in the systems on requirement. On the other hand, there are three major agency roles identified and furnished in the agents; diagnosis, scheduling and maintenance.

Diagnostic agent (DA) is developed to run the business model to identify if there is any disruption existing within the system, where the model is devised to check all the constraints if they are satisfied with provided data. The number of unsatisfactory constraints measures the level of disruptions existing within the system. Here, disruption can be defined as the gap between the values of running processes on the ground and the values strategically identified by the top management of the enterprise. DA records all the disruptions causing negative satisfaction across the entire enterprise. Meanwhile, the Scheduling agent (SA) checks the records by DA to verify if there is any diagnosed/realised disruption within the system, and then assigns relevant maintenance tasks to maintenance agent team, where the team can be made of identical agents, which are able to deliver all kind of maintenance. Depending on the design purpose of the agents, each member of maintenance agent (MA) team can be specialised in a particular maintenance and would be in charge of fixing corresponding disruptions only .

The complete MAS solver can work in the following logic, where the system is about hunting for the disruptions, and rectifying them accordingly. Let the business process model be denoted with $\mathcal{M}$, the list of disruptions diagnosed with $\mathcal{L}$ and the set of functionalities performed by the agents with $\mathcal{F}=\left\{f_{i} \mid i \in\left\{D A, S A, M A_{j}\right\}, j=1, . ., J\right\} . f_{D A}: \mathcal{M} \rightarrow$ $\mathcal{L}$ is defined to take the business model $\mathcal{M}$ as an input and to diagnose if there is any disruption within the system to generate the complete list, $L \subseteq \mathcal{L}$, and then, SA takes action to classify the complete list to be passed to corresponding MAs. SA's functionality is defined as the function to classify the list into sub-lists so that each can be assigned to corresponding MA as the task of maintenance, $f_{S A}: L \rightarrow T$, where $T=$ 
$\left\{T_{j} \mid j=1, \ldots, J\right\}$. Once each assigned, MAs take action to make the rectification in the systems so that the whole system to be inline with the enterprise strategy, $f_{M A_{j}}: T_{j} \rightarrow L_{j}^{*}$. The complete rectification in the system by MAs is the union of all results, defined as follows:

$$
L^{*}=\bigcup_{j=1}^{J} L_{j}^{*}
$$

Here, $L, L^{*}$ and $T$ are in matrix while $T_{j}$ and $L_{j}$ are in vector structures. The transition of the states of disruptions, which forms a cycle of actions by the system can be reflected as $L \Rightarrow T \Rightarrow L^{*}$, where the transition works in a cyclic process in which the system takes actions in cycles in interaction with the enterprise-wise corporate process.

\section{CAse Study: A CANCER-Care}

\section{A. Cancer-care systems}

Healthcare systems have complicated nature, with ever changing technology, new environmental pressures presenting almost daily, and complicated relationships among professionals, disciplines, departments, stakeholders, and organizations [17]. Cancer care as a subsystem of the overall healthcare system is one of the most complex ones. The domains of cancer care continuum span across several overlapping activities which start with prevention and risk reduction, an effective screening program, accurate and reliable diagnostic tests, coordination to optimise treatment modalities, survivorship surveillance and follow up and the availability of end of life care [18]. This is visualised in Fig. 4.

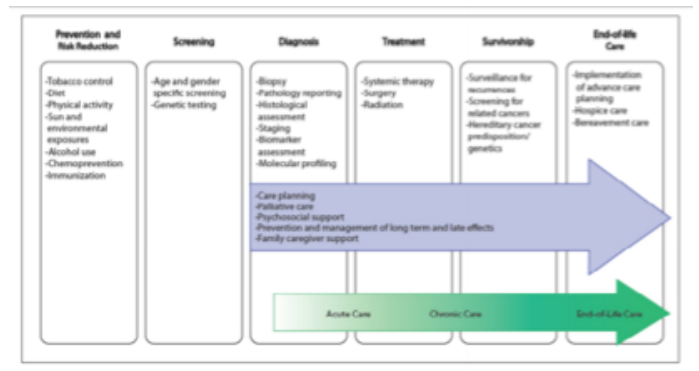

Fig. 4. The main domains of the cancer care continuum [18]

Improving healthcare especially cancer care is becoming increasingly significant, due to the individual human, economic and welfare of the society in general. Cancer is the second leading cause of death globally and is estimated to account for 9.6 million death in 2018 as the World Health Organization reports [19]. According to the global oncology trends 2017 advances, the estimated total worldwide global cost of cancer treatments of oncology and supportive care therapies increase to $\$ 113$ Billion in 2016 and the cost is increasing at an alarming rate of $11.6 \%$ [20], but unfortunately the literature is inconsistent regarding the relationship between cancer outcomes and health expenditure, some studies show that higher healthcare spending is not always associated with better cancer outcomes [21].
Brown et al [21] argues that it was not possible to establish a causal correlation between healthcare system characteristics and cancer outcomes. Further studies should explore in greater depth the associations between single health system factors and cancer outcomes, recognizing that in complex systems where context is all-important, it will be difficult to establish causal relationships. Better understanding of the interaction between healthcare system variables and patient and professional behavior may generate new hypotheses for further research. Tevaarwerk et al [22] recommends redesigning health care delivery systems by using Systems Engineering Initiatives for Patient Safety.

Cancer healthcare systems involve actors and tools at all levels the macro level (e.g., third party payers, electronic medical records), interpersonal dynamics (power structure, decision-making role preference), and individual personality differences and unique circumstances. They encompass all of the multi-level people, places, tools, organizational structures, and circumstances associated with the care of a patient, as well as the connections between them [23]. Information flow across the major components of a typical cancer care system is displeyed in Fig. 5.

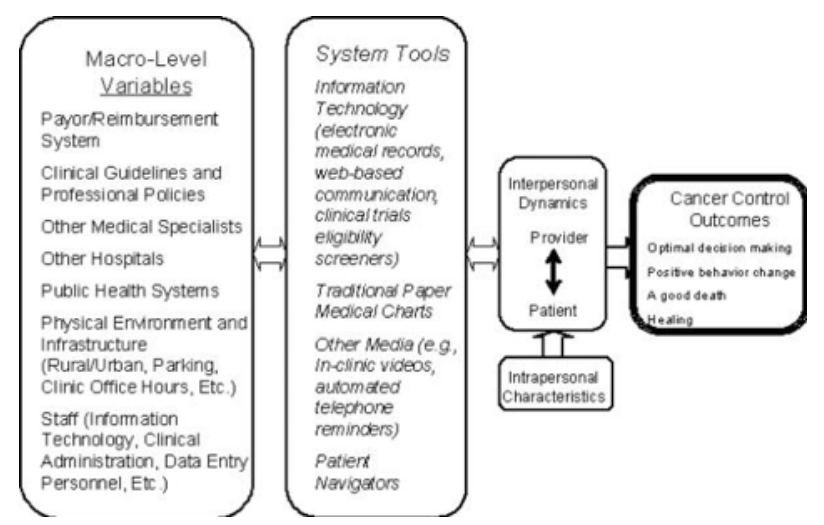

Fig. 5. A model for system communication in the cancer care health system [23]

There has seen an explosion of electronic information systems for hospitals, individual clinicians, and public health systems recently. These systems have not yet reached their potential, but they could facilitate rapid, accurate, and scalable data collection, data storage, data retrieval, and data sharing [24]. Such systems clearly have enormous implications for systems communication. For example, a state-of-the-art electronic system could facilitate intra-system communication of patient data, which provides a substrate for meaningful interpersonal communication among clinicians as well as between clinicians and patients. Improved medical data sharing could benefit individual patients. Another system-wide benefit could be improved tracking of public health problems through the collection of individual clinical data, instead of, as is often the case currently, waiting for physicians to complete paper reports and send them to public health departments (a process known to be slow, inconsistent, and incomplete) [25]. 
A systematic science of cancer communication research dissemination will likely require continued emphases on information technology tools, humanistic tools like patient navigators, and broad-based interdisciplinary approaches. Collaborations involving psychology, decision-making and behavioral science, and oncology will need to extend to include such disciplines as systems theory, classical communication theory, informatics, and organizational behavior [23].

Norberg and Cuming [26] defined Complex Adaptive Systems (CAS) as systems made up of interacting components (the system) whose interactions may be complex (in the sense of nonlinear) and whose components are diverse and/or have a capacity for learning that generates reactive or proactive adaptive behavior [23]. In this point of view, it can be considered as an CAS enterprise system with respect to size and complexity. There is a clear trend of growth in this industry upon the need and progress in care delivery and development.

\section{B. Exploration for implementing strategic alignment}

The discussion provided above confirms how large-scale the cancer care systems are, and how much they are subject to dynamic nature, which produce a constant change across the systems. A strategic approach must be adopted in order to govern cancer care systems and a change management becomes an unavoidable requirement in this regard. Following is a speculative scenario for demonstrating the need for strategic alingment and change management.

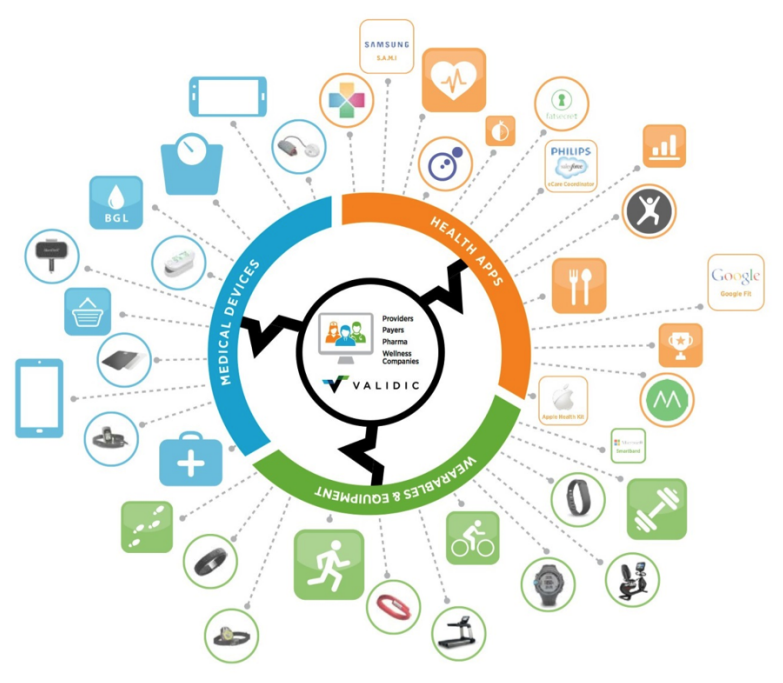

Fig. 6. A typical ecosystem for cancer care [27]

Fig. 6 demonstrates a cancer care ecosystem, which is delivered by an particular software solution offer by a vendor [27]. Three major components have been visualised with many links to possible sub-components, where can be treated as standalone systems. Looking into this figure itself gives insight into the characteristics of a cancer care system demonstrating how complex and scalable it can be. Since cancer care is a growing area and so much reliant upon ongoing research in various medical and IT disciplines, system updates and revisions in the principles and rules become inevitable. It requires a secure enforcement system to impose any change. The agent-based approach introduced above can be a prime candidate for implementation in this regard.

We assume that a cancer care system similar to Validic case visualised in Fig. 6, where the enterprise system is serving in a number of geographic sites, say 5 , with a number of interconnected in-house servers, say 1 server per site, facilitated with powerful compute and data clusters, say 2 data and 3 compute clusters. In addition, a number of cloud services are incorporated from various vendors. Assume that the system serves 10 thousand users in different capacities through a number of end-user applications, say 10 desktop, web and mobile applications. The configuration and maintenance of the system is managed on sites and coordinated from one centre.

Given above-mentioned circumstances, a multi agent system would be designed and developed to deploy in the backend of the enterprise system, where a diagnosis agent (DA) and a scheduler agent (SA) would be deployed on the coordinating site, while one maintenance agent (MA) would be installed and deployed per geographic site. Since all agents will act autonomously, DA will periodically review the status of the system and report to SA while SA will dispatch maintenance tasks to corresponding MA upon emerging changes.

This system will help coordinate all IT system across the enterprise in an automatic way without centrally coordinating the system for imposing any emerging change. It facilitates the configuration and change management of the entire system with reducing human reliance to a certain extent, which can be expand beyond strategic alignment towards daily maintenance of the system.

\section{Conclusions}

This position paper introduces an agent-based diagnosis and maintenance systems which includes a diagnostic model to health-check enterprise systems if it is strategicall fit, works incorporating with up-to-date corporate rules, principles and targets. It means to deliver strategic alignment in enterprise systems, which are large-scale software systems by nature, to discover deficiencies, inconsistencies and incompleteness across the enterprise. The diagnostic model embedded in DA agents helps reveal the gaps in between the strategic level and operational level on the ground while the rest of the agent-based system maintains and cures the discovered deficiencies. The approach is implemented for a cancer care case to demonstrate the proof-of-concept, where hypothetical assumptions are made to simulate the case.

The approch requires fine-tuning and further research to produce substantial results for feasibility of the idea and help reveal any missing aspects and prune any redundent components of the proposed framework. Further to that, the idea can be enhanced for incorporating with more configuration and maintenance coverage.

\section{REFERENCES}

[1] M. B. Ayhan, E. Öztemel, M. E. Aydin, and Y. Yue, "A quantitative approach for measuring process innovation: a case study in a manufac- 
turing company," International Journal of Production Research, vol. 51, no. 11, pp. 3463-3475, 2013.

[2] E. D. Morrison, A. K. Ghose, H. K. Dam, K. G. Hinge, and K. HoeschKlohe, "Strategic alignment of business processes," in International Conference on Service-Oriented Computing, pp. 9-21, Springer, 2011.

[3] M. Rashidirad, E. Soltani, and J. Syed, "Strategic alignment between competitive strategy and dynamic capability: Conceptual framework and hypothesis development," Strategic change, vol. 22, no. 3-4, pp. $213-$ 224, 2013.

[4] E. Damiani, F. Mulazzani, B. Russo, and G. Succi, "Saf: Strategic alignment framework for monitoring organizations," in International Conference on Business Information Systems, pp. 213-226, Springer, 2008.

[5] J. C. Henderson and N. Venkatraman, "Strategic alignment: a model for organizational transformation through information technology," Transforming organizations, pp. 97-117, 1992.

[6] D. Ivanov, "An adaptive framework for aligning (re) planning decisions on supply chain strategy, design, tactics, and operations," International journal of production research, vol. 48, no. 13, pp. 3999-4017, 2010.

[7] M. A. Shirazi and J. Soroor, "An intelligent agent-based architecture for strategic information system applications," Knowledge-Based Systems, vol. 20, no. 8, pp. 726-735, 2007.

[8] G. S. Kearns and R. Sabherwal, "Strategic alignment between business and information technology: a knowledge-based view of behaviors, outcome, and consequences," Journal of management information systems, vol. 23, no. 3, pp. 129-162, 2006.

[9] D. Avison, J. Jones, P. Powell, and D. Wilson, "Using and validating the strategic alignment model," The Journal of Strategic Information Systems, vol. 13, no. 3, pp. 223-246, 2004.

[10] L. Northrop, P. Feiler, R. P. Gabriel, J. Goodenough, R. Linger, T. Longstaff, R. Kazman, M. Klein, D. Schmidt, K. Sullivan, et al., "Ultra-large-scale systems: The software challenge of the future," tech. rep., Software Engineering Institute, Carnegie-Mellon University, Pittsburgh, PA, USA, 2006.

[11] I. Sommerville, D. Cliff, R. Calinescu, J. Keen, T. Kelly, M. Kwiatkowska, J. Mcdermid, and R. Paige, "Large-scale complex it systems," Commun. ACM, vol. 55, pp. 71-77, July 2012.

[12] M. E. Aydin and E. Öztemel, "Dynamic job-shop scheduling using reinforcement learning agents," Robotics and Autonomous Systems, vol. 33, no. 2, pp. $169-178,2000$.

[13] M. E. Aydin, "Metaheuristic agent teams for job shop scheduling problems," in Holonic and Multi-Agent Systems for Manufacturing, pp. 185-194, Springer, 2007.
[14] M. B. Ayhan, M. E. Aydin, and E. Öztemel, "A multi-agent based approach for change management in manufacturing enterprises," Journal of Intelligent Manufacturing, vol. 26, no. 5, pp. 975-988, 2015.

[15] M. E. Aydin, "Coordinating metaheuristic agents with swarm intelligence," Journal of Intelligent Manufacturing, vol. 23, pp. 991-999, Aug 2012.

[16] M. E. Aydin and R. Fellows, "Building collaboration in multi-agent systems using reinforcement learning," in International Conference on Computational Collective Intelligence, pp. 201-212, Springer, 2018.

[17] C. E. Duquette and Q. Solutions, $Q$ Solutions. Essential Resources for the Healthcare Quality Professional, ch. Leadership and Management. National Association for Healthcare Quality (NAHQ), US, 2012. 3rd ed.

[18] L. A. Levit, E. Balogh, S. J. Nass, P. Ganz, et al., Delivering high-quality cancer care: charting a new course for a system in crisis. National Academies Press Washington, DC, 2013.

[19] W. H. Organization, "World health organization/cancer." http://www. who.int/cancer/en/. Accessed 3rd Oct 2018

[20] I. Institue, "Global oncology trends 2017: Advances, complexity and cost," tech. rep., IQVIA Institue, 2017.

[21] S. Brown, M. Castelli, and D. Hunter, "How might healthcare systems influence speed of cancer diagnosis: A narrative review," Social Science and Medicine, vol. 116, pp. 56-63, 2014.

[22] A. J. Tevaarwerk, J. R. Klemp, G. J. Londen, B. W. Hesse, and M. E. Sesto, "Moving beyond static survivorship care plans: A systems engineering approach to population health management for cancer survivors," Cancer, September 2018.

[23] J. Quillin, K. Tracy, and J. Ancker, "Healthcare system approaches for cancer patient communication," Journal of Health Communication, vol. 14, no. 1, pp. 85-94, 2009.

[24] R. Kukafka, J. Ancker, C. Chan, J. Chelico, S. Khan, S. Mortoti, K. Natarajan, K. Presley, and K. Stephens, "Redesigning electronic health record systems to support public health," Journal of Biomedical Informatics, vol. 40, no. 4, pp. 398-409, 2007.

[25] T. P. H. W. Group, "Connecting for health: A public-private collaborative," tech. rep., The Markle Foundation, July 2003.

[26] J. Norberg and G. Cumming, Complexity theory for a sustainable future. Columbia University Press, 2008.

[27] D. Schiller, "Validic bridges the healthcare mobile data interoperability gap." http://sjfventures.com/case-study/validic-bridges-thehealthcare-mobile-data-interoperability-gap/. Accessed September 13, 2018. 